Acta Poetica 25-1

PRIMAVERA

2004

\title{
Glosas: ¿lenguas del texto o malas lenguas? Lexicógrafos, trasladadores y declaradores de textos en el Siglo de Oro
}

\begin{abstract}
Ana Castaño
Prestando especial atención al siglo XVII en España, se revisan someramente algunos de los significados que la palabra glosa ha tenido a partir de sus primeras documentaciones en nuestra lengua, y se señala una de las acepciones que han sido más persistentes a lo largo de los siglos y en diferentes lenguas europeas. También se apunta el camino que va, por un lado, de la glosa a la definición y, por el otro, de la glosa a la traducción. De paso, se da noticia de algunos pasajes que Benito Remigio Noydens, editor y "glosador" del Tesoro de Covarrubias tomó de un glosario de Alejo Venegas. Por último se comenta —o se glosa— brevemente un poema de Seamus Heaney titulado precisamente "The first gloss", así como la traducción al español que hizo Pura López-Colomé.

Focusing mainly on the seventeenth century in Spain, some of the meanings of the word gloss are quickly reviewed here, starting form its first documentations in Spanish. One of these meanings, it is pointed out, is particularly recurrent through different centuries and different European languages Also, a line is traced that goes from the idea of gloss to that one of definition, on one hand; and to the idea of translation, on the other. In passing, notice is given of certain borrowings made by Benito Remigio Noydens, editor and "glosador" of Covarrubias' Tesoro, of a previous glossary by Alejo Venegas. And, last, a brief comment -or gloss- is made to a poem by Seamus Heaney ("The first gloss") as well as to its translation to Spanish by Pura López-Colomé.
\end{abstract}




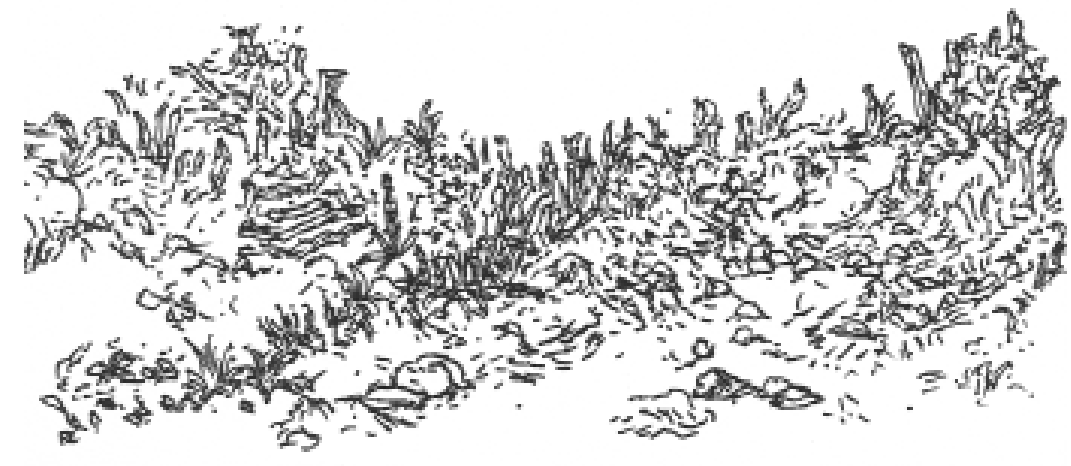


Acta Poetica 25-1

PRIMAVERA

2004

\section{Ana Castaño}

Glosas: ¿lenguas del texto o malas lenguas? Lexicógrafos, trasladadores y declaradores de textos en el Siglo de Oro

El Tesoro de la lengua castellana o española (1611), de Covarrubias, comienza su definición de "glosa" señalando el origen griego de esa palabra ( $g$ losa = 'lengua'), y a continuación explica: "comúnmente se toma por las anotaciones y comentos que declaran los textos o otra cualquier escritura, por quanto son como lenguas e intérpretes. Glossar alguna cosa escrita o dicha es interpretarla". Y en seguida otra entrada dice: "glossar las palabras; vulgarmente es darles otro sentido del que suena y a veces del que pretendió el que las dixo".

Tenemos entonces que ya en 1611 contábamos con una buena definición del término ( glossa y glossar), que registraba las dos principales acepciones que habrían de perdurar en nuestra lengua hasta la más reciente edición del Diccionario de la Real Academia Española (2001): una positiva, según la cual glosar es 'aclarar' 'explicar', 'interpretar'; y otra de carga negativa, que define esta palabra como 'malinterpretar deliberadamente'. 1

\footnotetext{
${ }^{1}$ La tercera acepción que da el DRAE para glosar es: "Interpretar y tomar en mal sentido y con intención siniestra una palabra, una proposición o un acto". El Diccionario de Autoridades [Madrid, 1732], segunda acepción, traía: “...interpre-
} 


\section{Claro está que, mucho antes de ser incluida en los dicciona- rios, la acepción negativa de glosa había sido conocida y utili- zada por los escritores. Abundan los ejemplos en la literatura europea medieval y renacentista. ${ }^{2}$}

tar, o tomar a mala parte y con intención siniestra alguna palabra o proposición". En el Diccionario castellano con las voces de ciencias y artes y sus correspondencias en francés, latín e italiano en cuatro volúmenes [Madrid, 1786-93], de Esteban de Terreros y Pando (1707-1782), es donde más atenuada he encontrado la acepción negativa. Únicamente en la entrada glosador, se da como primera acepción la de "censor, crítico", y en glosar, tercera acepción, "comentar, criticar, acriminar". Es de notarse que Terreros y Pando es el único que registra para glosa la acepción (clásica y más etimológica) de "interpretación o traducción que se hace, palabra por palabra, de un autor u obra en otra lengua"; y, como extensión de ésta, incluye una segunda acepción: "Se toma también por la interpretación que se da a un autor aunque sea en la misma lengua y de aquí se dice de la que se hace de cualquier palabra que se oye, etc. y en este sentido significa crítica, comentario, interpretación, nota."

2 En España, Juan Ruiz incluye el término como una de tantas maneras de llamar a la alcahueta: "A la tal mensajera nunca le digas maca,/Byen o mal que gorgee, nunca l'digas pycaça,/ Señuelo, cobertera, almadana, coraça,[...]/ Aguijón, escalera, nin abejón nin losa,/ Traylla nin trechón nin registro nin glosa:/ Desir todos sus nonbles es a mí fuerte cosa,/ Nonbles e maestrías más tyenen que raposa" (Libro de buen amor, 927b); Pero López de Ayala, en el Rimado de palacio: "Non vos querrýa dezir en aquesto/ salvo verdat et non otra cossa,/ nin vos querrýa mentyr en el texto,/ nin por apostila poner otra glossa:/ aquesta sentençia assý como rossa/ la tengo yo por difinitiva..." (Apéndice 1, fragmento C, 7 , ed. de Michel García, Madrid, Gredos, 1978, vol. II, 381); Francisco Delicado, al final de La lozana andaluza: "Y si alguno quisiere decir que hay palabras maliciosas, digo que no quiera nadie glosar malicias imputándolas a mí, porque yo no pensé poner nada que no fuese claro a ojos vistas... (ed. de Claude Allaigre, Madrid, Cátedra, 1985, 485: agradezco este dato a mi colega Tatiana Bubnova). Con estas acepciones negativas, glosar está presente también en la literatura medieval y renacentista de Inglaterra y Francia. En Chaucer por ejemplo, con el sentido de 'manipular' 'distorsionar algo a favor de uno': "Glosynge is a glorious thyng, certeyn, For lettre sleeth, so as we clerkes seyn" (The Canterbury Tales, "The Summoner's Tale", v. 1794); 'embaucar' 'engañar con palabras': "And therwithal so wel koude he me glose, Whan that he wolde han my belle chose"("The wife of Bath's Prologue"); o: "Of me certeyn, thou shalt nat been yglosed." ("The Manciple's Prologue"). El Dictionary of the English Language de Samuel Johnson (1755) trae varios ejemplos posteriores: "If then all souls, both good and bad, do teach,/ with gen'ral voice, that souls can never die;/ 'Tis not man's flatt'ring gloss, but nature's speech,/ Which, like God's oracles, can never lie" (Davies); "He seems with forged quaint conceit/ To set a gloss upon his bad intent" (Shakespeare, Henry VI); "Poor painters oft with silly poets join,/ to fill the world with strange but vain conceit;/ one brings the stuff, the other stamps the coin,/ Which breeds nought else but glosses of deceit" (Sidney). En cuanto al 
En la siguiente entrada (glosadores) registrada en el Tesoro, después de una sobria y correcta definición ("los que interpretan glosando"), nos sorprende una glosa de carne y hueso, introducida por el jesuita Benito Remigio Noydens en su edición del Tesoro de 1673. La relativa extensión y la carga emotiva de esta glosa contrastan con el carácter escueto e imparcial de las definiciones de glosa que nos había dado Covarrubias. Efectivamente, se trata de una observación de varias líneas (escrita al margen de una definición de sólo cuatro palabras), que continúa y enfatiza la acepción negativa del término. Vale la pena reproducirla completa:

[Ya dixo el autor que la glossa es la lengua del texto; yo digo que assí como la glossa es la lengua del texto, assí ocasional y accidentalmente la copia demasiada de glossas ha sido enmudecimiento de lenguas y aterramiento de ingenios. De donde vemos por experiencia que cuando se usava el proverbio que dize: liber librum aperit, que un libro es glossa de otros, sabían mucho más los hombres que agora, que con confiança de glossas, comentos, annotaciones, escolias, observationes, castigationes, miscellaneas, centurias, paradoxas, collectaneas, lucubrationes y additiones, han dexado ranciar los ingenios y enmudecerse las lenguas, y, lo que peor es, por la multiplicación de las glossas están ahogados los textos de leyes y medicina, con que se pierden las haziendas de los pleyteantes y se multiplican las enfermedades de los achacosos, y será mucho si no se ensanchan las conciencias.]

Las críticas a la prolijidad de glosas y comentarios no eran ninguna novedad en el Siglo de Oro, pero siempre pensé que este efusivo ataque, al igual que otras tantas glosas y adiciones

francés, en el Roman de la rose aparece una acepción muy similar de glosar ('maquillar', 'encubrir'), como cuando una doncella dice que no pecará si nombra las partes nobles del cuerpo "Par plain texte sanz mettre gloses..." (v. 6954; agradezco este dato a Cristina Azuela). También en el italiano está presente el matiz negativo de glosar, como veremos más adelante. 
de Noydens al Tesoro, había salido originalmente de su pluma. Además, a pesar de que sus intervenciones fueron alguna vez calificadas como "de pésimo tino y peor gusto", ${ }^{3}$ llegué a preguntarme si no habría escrito justamente esa larga glosa en contra de las glosas con intención irónica, es decir, con plena conciencia de estar dando un buen ejemplo precisamente de lo que quería criticar. De cualquier manera, un día, hojeando el "glosario" que Alejo Venegas añade en 1543 a una obra piadosa que había escrito él mismo unos años antes (La Agonía del tránsito de la muerte, Toledo, 1537), ${ }^{4}$ en la entrada correspondiente a glosa me encontré con el pasaje que acabo de citar, y pude constatar que Noydens lo había reproducido casi exactamente en su edición del Tesoro de Covarrubias sin citar la fuente (como, por otra parte, encontré que hizo con muchos otros pasajes de este glosario de Venegas). ${ }^{5}$

$\mathrm{Si}$ ya resultaba curioso que el editor de un lexicógrafo - $\mathrm{O}$ "glosógrafo", como se les llamó en la Antigüedad— publicara semejante ataque al gremio, llamaba más la atención aún que este ataque a las glosas formara parte de un glosario anterior, escrito por otro "glosógrafo". ¿Por qué este repetido empeño en la autocrítica? Más allá de constituir un tópico, parece como si los glosadores, al introducir ese tipo de reflexiones sobre los excesos de su oficio, quisieran quedar curados de este "achaque de glosas"6 aun a sabiendas de que, por defini-

\footnotetext{
${ }^{3}$ En el breve artículo que dedica la Enciclopedia ESPASA (1958) a Sebastián de Covarrubias.

${ }^{4}$ La Breve declaración de las sentencias y vocablos obscuros que en el libro del Tránsito de la Muerte se hallan. Escripta por el mismo autor [1543], e incluida junto con la Agonía... en la edición de Miguel Mir, Escritores místicos españoles, t. 1, Madrid, 1911 (N.B.A.E., t. XVI).

${ }^{5}$ Una simple búsqueda superficial comprueba que son varios los fragmentos que Noydens tomó del glosario de Alejo Venegas. Véanse por ejemplo las entradas: mamona (en Covarrubias véase rico), polilla, poros, refrán, sazón, simón (en Covarrubias ver simonía), talento, etc.

${ }^{6}$ Cervantes atribuye a Don Quijote cierto "achaque de glosas" (refiriéndose a las glosas líricas, procedimiento poético muy popular en aquellos años) cuando, hablando con el estudiante poeta en casa del "Caballero del Verde Gabán”, le pre-
} 
ción, era inherente a su actividad. El caso de Venegas, bastante anterior como hemos visto y menos conocido como lexicógrafo, resulta más elocuente todavía por la cantidad de reflexiones que nos dejó sobre su oficio y su método, que revelan una aguda conciencia crítica de su propia labor. ${ }^{7}$

$* * *$

Pero volvamos a la acepción positiva de glosa, la que la define como "lengua o intérprete". Así vista, la glosa puede consistir en una definición, una explicación, una interpretación o incluso la traducción de un término difícil o especializado a la lengua común.

En los preliminares de su glosario, Venegas propone algunos deslindes y definiciones para establecer con claridad qué es "declaración" (definición). Establece primero las partes de ésta: explanación, dilatación, contracción e interpretación. Divide a su vez la explanación en glosa, entimema y escolio; la dilatación, en cuento poético y paráfrasis; la contracción, en comentario, epítome y detruncación; y la interpretación (o traducción), en traslación de palabra por palabra y en traslación de sentido por sentido. Hace después una distinción - que resulta original aunque poco convincente - entre comentario y glosa, en la que, de paso, se deja sentir quizás un débil eco de aquella acepción negativa del término: el comentario es explicación de las cosas reales, y la glosa, de las imaginarias. Define después la paráfrasis como una mezcla de texto y glosa, "porque el texto sirve de huesos y la glosa de carne, de modo

gunta: “¿qué versos son los que agora trae entre manos...? Y si es alguna glosa, a mí se me entiende algo de achaque de glosas, y holgaría saberlos..." (Quijote II, 18).

${ }^{7}$ Véanse, además de los preliminares de su glosario a que me refiero en seguida, las continuas reflexiones que se encuentran dispersas a lo largo de la obra, por ejemplo: “...se excusasen muchas rebueltas de pleitos en las repúblicas si estuviesen por ley tasadas é limitadas las significaciones de los vocablos” (288). 
que resulte un cuerpo de entram[b]os" (261). Luego nos da su versión de lo que debería ser la "interpretación"o "traslación" (es decir, la traducción): "El que traslada: In principio erat verbum por "en el principio era la palabra", no mira que en este lugar se toma principio por el Padre eterno, y verbum por la noticia y conoscimiento que eternalmente el Padre tiene de sí, y aquel verbo erat no significa tiempo, sino el ser eterno de la segunda persona de la Sanctísima Trinidad...". Para concluir, Venegas nos dice que en la presente obra utilizará "todas estas diferencias y especies de declaración" (262). Como podrá imaginarse, el "diccionario" — o "declaración de vocablos"que viene a continuación, lejos de estar libre de glosas, será un conjunto de definiciones, etimologías, glosas y material folklórico, didáctico, moralizante y erudito muy semejante, en pequeño, al Tesoro de Covarrubias. Y todo esto a pesar de esa invectiva, ya citada, en contra de los excesos de glosas.

¿Dónde están los límites, entonces, entre glosa y "declaración" (glosa y definición), o entre glosa y traducción? En los Siglos de Oro, por lo menos, eran muy confusos. Y en el nuestro siguen siéndolo. Hay glosas (hoy casi siempre en forma de notas a pie de página) que consisten en la definición escueta de un término; y sigue habiendo entradas de diccionario que contienen mucho más que la definición de un término (juicios y opiniones personales además de ejemplos y "autoridades", etc). Por otra parte, en el diccionario de Venegas, como sucede en muchos otros textos de la Edad Media y del Siglo de Oro, la traducción es considerada explícitamente como una de las formas de la "declaración", al mismo nivel que otras de estas formas (con las cuales se confundía muchas veces): las glosas, los comentarios y demás maneras de hacer accesibles a un público más amplio textos que de otra forma hubieran resultado muy difíciles o incomprensibles para la gran mayoría. Recordemos que ya en pleno siglo XVIII, Esteban de Terreros y Pando daba como primera acepción de glosa precisamente la 
acepción etimológica de 'traducción', y después procedía a deslindar entre traducción a otra lengua y traducción dentro de una misma lengua (ver nota 1 ).

Por lo que hemos visto, las diferentes acepciones de la palabra glosa se pueden dividir en dos grandes rubros: las "positivas" ('lengua del texto') y las "negativas" ('mala lengua'). Y esta ambivalencia no se da sólo en el español. En nuestros días sigue igualmente presente al menos en el inglés, el italiano y el francés ${ }^{8}$ (y probablemente en muchas otras lenguas). Voy a detenerme un momento en el inglés, donde ha ocurrido una interesante complicación en la historia de este término.

Tanto el diccionario de Samuel Johnson, del siglo XVIII, como el Webster's del siglo xx (1983) registran, junto con las acepciones "positivas" ("A scholium, a comment" en Johnson; "words of explanation or translation...", "A note or comment of explanation... as in a footnote or a margin" en el Webster's), las "negativas" ("An interpretation artfully specious" en Samuel Johnson; "a false or misleadig explanation" en el Webster's).

\footnotetext{
${ }^{8}$ En lo relativo al italiano y al francés modernos, el Grande dizionario della lingua italiana de Salvatore Battaglia (Torino, Unione tipografico-editrice torinese, 1961-2002) en la entrada glossa registra, después de las acepciones tradicionales de 'nota o comentario explicativo o interpretativo', la siguiente: "Discorso ozioso e inutile, chiacchiera superflua", y entre las locuciones: "Fare la glossa: dare giudizi negativi, criticare, atteggiarsi a giudice". En la entrada glossare da, como tercera acepción (figurativa): "Vagliare; esprimere giudizi, valutare, commentare (e per lo piú indica una disposizione d'animo poco benevola)"; y lo mismo en la locución: "Glossare il testo a proprio vantaggio: travisare volontariamente una disposizione o una situazione per volgerla a proprio favore". El Dizionario etimologico dei vocaboli italiani di origine ellenica, de M. A. Canini (Torino, Unione tipografico-editrice torinese, 1925) registra, también en tercer lugar, la siguiente acepción: "Giunta, al racconto veridico di un avvenimento, di circostanze inventate e di asseverazioni critiche, in senso maligno anzi che no". El Nouveau Petit Robert (Paris, Dictionnaires Le Robert, 1993) registra, como segunda acepción de GLOSE: "Commentaire oiseux ou malveillant. Les gloses des bavards, des commëres". Y en la entrada GLOSER, la segunda acepción es: "Se perdre en discussions, en vains discours à propos de tout".
} 
Por añadidura, el Webster's registra no una sino dos entradas para gloss. La otra (que aparece en primer lugar) es un homónimo procedente del antiguo escandinavo gloa> gloss, 'brillo', con el significado de: "brightness or luster of a smooth surface", pero cuya segunda acepción es: "a specious appearance or representation; external show that may deceive". 9 Este otro gloss (<gloa) viene pues a complicar — o a enriquecer — la vida del término de origen griego en la lengua inglesa.

La Encyclopaedia Britannica, en su decimoprimera edición (1911), se pregunta por el origen de la acepción negativa (que llama "usual") de esta palabra, y propone atrubuirlo a la historia de la glosa literaria "propiamente dicha". Además, observa que la acepción negativa de gloss ("an interpretation, especially in a disingenuous, sinister or false way"), así como la forma gloze (simple variante ortográfica, asociada específicamente a la idea de paliar o adornar algo), podían haberse cruzado semánticamente con ese otro gloss (del antiguo escandinavo 'brillo'), influyendo en su segundo significado ('mera apariencia externa, barniz'). ${ }^{10}$ Efectivamente, habrá que indagar en la historia de la glosa literaria para encontrar quizás una explicación de por qué las lenguas del texto se rodearon, desde muy pronto, de un halo de malicia para convertirse en malas lenguas. Claro está que habrá que tomar en cuenta la naturaleza

\footnotetext{
${ }^{9}$ Webster's New Twentieth Century Dictionary, 2a ed., New York, Dorset \& Baber, 1983. Johnson registra sólo una entrada para gloss (<griego, a través del francés) pero, con el número 3, incluye la acepción correspondiente al gloss escandinavo: "Superficial lustre". Sin embargo, los ejemplos que da no refuerzan esa idea de "superficial".

10 "The history of the literary gloss in its proper sense has given rise to the common English use of the word to mean an interpretation, especially in a disingenuous, sinister or false way; the form gloze, more particularly associated with explaining away, palliating or talking speciously, is simply an alternative spelling. The word has thus to some extent influenced, or been influenced by, the meaning of the etymologically different "gloss"= 'lustrous surface' (from the same root as glass; cf. glow), in its extended sense of 'outward fair seeming'. (The Encyclopaedia Britannica, decimoprimera edición, New York, Cambridge University Press, 1911, vol. XII, 124, n. 1).
} 
de la actividad del glosador, cuyo trabajo transcurre literalmente al margen de la "obra" y siempre, por así decirlo, en la resistencia. En cierto sentido, escribirle glosas a un texto es como sembrarlo de minas o colocarse a sí mismo en la posición del francotirador.

Pero la persistencia de la acepción negativa de glosa, tanto en inglés como en español, tiene también sus contraejemplos. Hace años me encontré con uno. Se trata de un breve poema en inglés del poeta irlandés Seamus Heaney, y de su traducción al español hecha por Pura López Colomé. Reproduzco primero el poema y una versión literal:

\section{"The First Gloss" \\ Take hold of the shaft of the pen \\ Subscribe to the first step taken from a justified line into the margin. ${ }^{11}$}

["La primera glosa" Toma con firmeza el mango de la pluma/ suscribe el primer paso dado/ desde una línea justificada/ hasta invadir el margen].

No hay aquí el menor rastro de la idea de glosa como comentario malévolo o distorsión deliberada. Muy por el contrario, este breve poema es, entre otras cosas, una apología de la glosa como "lengua del texto". Se trata de un homenaje a la labor de ese hipotético primer copista de la antigüedad que se aventuró a ir más allá del texto "justificado" para escribir su glosa en los márgenes de la página que estaba copiando. Tam-

${ }^{11}$ Station Island [London, 1984] New York, Farrar-Strauss-Giroux, 1995, 97. 
bién es una invitación, expresada en imperativos y dirigida quizás a todos nosotros, a seguir ese primer ejemplo e ir siempre, cuando leemos y cuando escribimos, más allá de lo ya dado, del texto "justificado", para llegar a donde podamos finalmente decir lo nuestro. Vistos así, estos cuatro versos con su título constituyen un arte poética en miniatura.

La ausencia total de aquella acepción negativa en el poema de Heaney es significativa. Pero resulta también significativa la vuelta de tuerca debida a la traductora al español, que eliminó del poema la idea de 'glosa' y tradujo el título como "El primer resplandor". Con ello ha ocurrido, ahora sí, un cruce de palabras en el que domina la más "brillante" de las dos entradas para la palabra gloss que da el diccionario Webster's (y, de paso, la opción que resulta más sonora en nuestra lengua). Veamos la traducción del poema completo

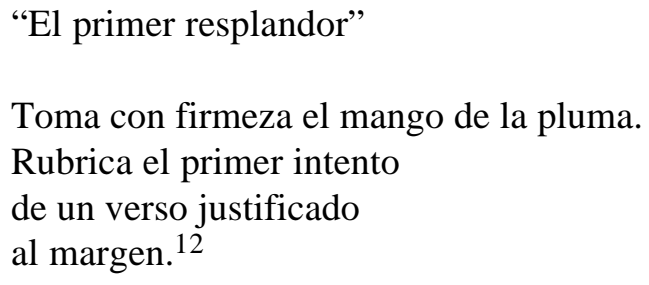

En su conjunto, la versión española no habla ya del primer lector o amanuense que se atrevió a escribir una glosa al margen. En cambio, centra la atención del poema en la actividad del poeta (los versos "justificados al margen"), a la que parece rodear de cierto halo mistificador. El hecho es sintomático. Se diría que, tras haber sido de muchas formas vituperado, el anónimo trabajo de los glosadores antiguos parece haber sido fi-

12 Isla de las estaciones, traducción de Pura López-Colomé, México, Ediciones Toledo, 1991, 111. 
nalmente olvidado. Y sin embargo... ahí está el homenaje que le rinde a la primera glosa uno de los mejores poetas de nuestro tiempo. En cuanto a las notas que anteceden, el lector podrá decidir si los breves comentarios, ejemplos y contraejemplos aquí aducidos funcionan efectivamente como lenguas del texto, o si simplemente han desempeñado el papel de malas lenguas. 